\title{
Electron-Hole Interference in an Inverted-Band Semiconductor Bilayer
}

\author{
Matija Karalic $\odot,{ }^{1,},{ }^{*}$ Antonio Štrkalj $\odot,{ }^{2, \S}$ Michele Masseroni, ${ }^{1}$ Wei Chen $\odot,{ }^{2,3, \dagger}$ Christopher Mittag, ${ }^{1}$ Thomas Tschirky, ${ }^{1}$ \\ Werner Wegscheider $\odot,{ }^{1}$ Thomas Ihn $\odot,{ }^{1, \$}$ Klaus Ensslin $\odot,{ }^{1}$ and Oded Zilberberg $\odot^{2}$ \\ ${ }^{1}$ Solid State Physics Laboratory, ETH Zurich, 8093 Zurich, Switzerland \\ ${ }^{2}$ Institute for Theoretical Physics, ETH Zurich, 8093 Zurich, Switzerland \\ ${ }^{3}$ National Laboratory of Solid State Microstructures and Department of Physics, \\ Nanjing University, Nanjing 210093, China
}

(Received 13 November 2019; revised 13 February 2020; accepted 29 April 2020; published 9 July 2020)

\begin{abstract}
Electron optics in the solid state promises new functionality in electronics through the possibility of realizing nano- and micrometer-sized interferometers, lenses, collimators, and beam splitters that manipulate electrons instead of light. Until now, however, such functionality has been demonstrated exclusively in one-dimensional devices, such as in nanotubes, and in graphene-based devices operating with $p$ - $n$ junctions. In this work, we describe a novel mechanism for realizing electron optics in two dimensions. By studying a two-dimensional Fabry-Perot interferometer based on a resonant cavity formed in an InAs/GaSb double quantum well using $p$ - $n$ junctions, we establish that electron-hole hybridization in band-inverted systems can facilitate coherent interference. With this discovery, we expand the field of electron optics in two dimensions to encompass materials that exhibit band inversion and hybridization.
\end{abstract}

DOI: 10.1103/PhysRevX.10.031007

\section{INTRODUCTION}

Common interferometers in optics, such as Fabry-Perot or Mach-Zehnder interferometers, rely on the interference of monochromatic waves with the same propagation direction. In this sense they can be regarded as one dimensional. In an optical Fabry-Perot interferometer, the interference pattern can be observed as a periodic change of maxima and minima in the transmitted intensity while the wavelength of the light is gradually varied. However, the wavelength period of the interference pattern depends on the incident angle of the light because it is the projection of the wave vector onto the interferometer axis that enters the conditions for constructive and destructive interference. As a result, the interference pattern is averaged out if light of all possible incident angles is sent through the interferometer at once. The same arguments apply to electronic Fabry-Perot interferometers. Pronounced interference is usually observed only in one-dimensional systems, such as carbon nanotubes

\footnotetext{
*makarali@phys.ethz.ch

†pchenweis@gmail.com

\#ihn@phys.ethz.ch

${ }^{\S}$ These authors contributed equally to this work.
}

Published by the American Physical Society under the terms of the Creative Commons Attribution 4.0 International license. Further distribution of this work must maintain attribution to the author(s) and the published article's title, journal citation, and DOI.
Subject Areas: Electronics, Mesoscopics, Topological Insulators

[1] or quantum Hall edge states [2,3], where the propagation direction of electrons is restricted to one dimension.

Recently, electronic interference has been discovered in two-dimensional $p n p$ junctions (or $n p n$ junctions) of single- and multilayer graphene [4-6], where electrons (or holes) are incident under all possible angles. There, Klein or anti-Klein tunneling in conjunction with Dirac's spectrum leads to a specific selectivity for incident angles that enables the observation of interference in these twodimensional geometries without the need for additional confinement in the transverse direction. Together with the realization of electron lenses, collimators and beam splitters [7-12], this discovery has marked a leap forward for electron optics in the solid state.

Here, we report an interference mechanism fundamentally different from that in graphene by studying transport through a two-dimensional Fabry-Perot interferometer realized using $p-n$ junctions in an inverted $\mathrm{InAs} / \mathrm{GaSb}$ double quantum well. The interference is facilitated by electron-to-hole scattering in the band-inverted regime. Such a scenario does not filter the number of participating transport channels, but instead produces many parallel onedimensional channels that share almost identical conditions for constructive interference. This leads to a nonvanishing interference pattern even after angle averaging. With this finding, we expand the field of electron optics in two dimensions to include materials that exhibit band inversion and hybridization. 


\section{DEVICE DESCRIPTION}

Figure 1(a) is a false-color scanning-electron micrograph showing an enlargement of a typical device used in this work. The device is defined by an etched mesa and a combination of overlapping metallic gates, see Fig. 1(b), placed on top of a semiconductor heterostructure hosting the coupled InAs and GaSb quantum wells. The nominally undoped heterostructure is grown using molecular-beam epitaxy along the [100] crystallographic axis on a GaSb substrate. However, unintentional $n$-type doping characteristic of our heterostructures leads to an excess of electrons in the absence of any externally applied electric fields [13]. The charge carriers are confined in two dimensions to the planes of the buried quantum wells, which extend in $x-y$ direction and have thicknesses in $z$ of 13.5 (InAs) and $8 \mathrm{~nm}(\mathrm{GaSb})$, respectively.

To fabricate devices [14], we initially define the mesa of width $W=5 \mu \mathrm{m}$ using wet chemical etching. Then, we deposit the first layer of $\mathrm{Al}_{2} \mathrm{O}_{3}$ which serves as an insulating oxide layer. Afterwards, we evaporate the metallic local gates, followed by depositing the second layer of $\mathrm{Al}_{2} \mathrm{O}_{3}$. Finally, we evaporate the large-area, or global, metallic top gate before depositing metal to define Ohmic contacts to the buried quantum wells. The local gates span the full device width and are electrically separated from the heterostructure surface below and the top gate above by the insulating oxide layers. Below a local gate, the density of charge carriers in the quantum wells is affected by this gate only. The adjoining outer regions (leads) are affected by the top gate exclusively [see the Supplemental Material for a full electrostatic simulation [14]]. A typical device contains multiple local gates of varying lengths, allowing us to study the length dependence of the interference in the same device. The image in Fig. 1(a) is an enlargement of the region in the vicinity of a single local gate of lithographic length $L=320 \mathrm{~nm}$.

We commonly inject a de current $I \sim 100 \mathrm{nA}$ into the device at $1.3 \mathrm{~K}$ and record the resulting junction voltage $V_{j}$ as a function of the voltage applied to the top gate $V_{\mathrm{TG}}$ and the voltage applied to the local gate $V_{\mathrm{LG}}$ [Figs. 1(a), 1(b)]. In order to probe the locally gated region directly, we also apply a small ac modulation to the local gate $\delta V_{\mathrm{LG}}$ and pick up the corresponding ac response in the junction voltage $\delta V_{j}$. Then, $\delta V_{j} / \delta V_{\mathrm{LG}} I$ represents the quantity $d R_{j} / d V_{\mathrm{LG}}$ and is sensitive solely to changes in the transmission of the locally gated region. From now on, for reasons to become apparent shortly, we will refer to the locally gated region as the "cavity."

\section{EXPERIMENTAL RESULTS}

In the InAs/GaSb bilayer system, which has recently been studied due to its topological insulator properties [15-18], electronlike and holelike states coexist in the energetic interval characterized by band inversion [19-22]. Here, we employ the term "bilayer" to refer to the InAs and GaSb quantum wells which form a bipartite, or bilayer, system when considered as a whole. In the region of band inversion, a hybridization gap opens in the in-plane dispersion due to electron-hole coupling in the form of a tunnel coupling between the adjacent quantum wells. The charge neutrality point (CNP) resides within the hybridization gap, marking the point at which the system is overall charge neutral, as the
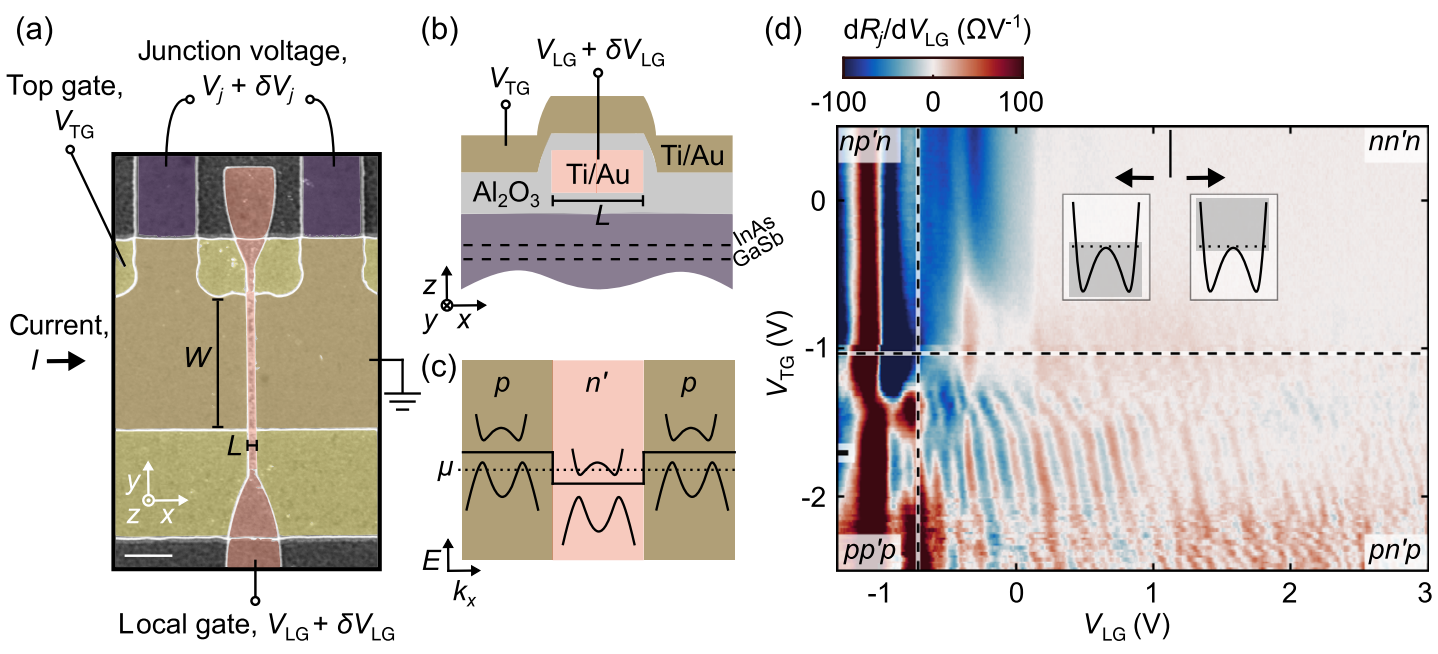

FIG. 1. (a) False-color image describing the device structure and operation. The scale bar represents $2 \mu \mathrm{m}$. (b) Schematic device cross section. (c) Exemplary band alignment $E(k)$ in the $p n^{\prime} p$ configuration; $\mu$ is the electrochemical potential, indicated by the dotted line, and the solid line indicates the charge neutrality point (CNP) in each region. (d) Color map depicting the differential junction resistance $d R_{j} / d V_{\mathrm{LG}}$ as a function of $V_{\mathrm{LG}}$ and $V_{\mathrm{TG}}$ at $1.3 \mathrm{~K}$. The resulting phase diagram is subdivided into quadrants according to the chargecarrier configuration. The horizontal and vertical dashed lines mark the CNPs of the leads and the cavity, respectively. The insets show the parts of the band structure probed to the left and right of the solid line at $V_{\mathrm{LG}}=1.1 \mathrm{~V}$ in the $p n^{\prime} p$ configuration, as highlighted by the shading. 
nonvanishing electronlike and holelike charges compensate each other exactly. The hybridization gap is typically several milli-electron volts in magnitude [17,20,22].

To explore the available parameter space, we tune the voltages $V_{\mathrm{TG}}$ and $V_{\mathrm{LG}}$ to obtain a variety of charge-carrier configurations in the device. We label the configurations with $\left(x, y^{\prime}, x\right)$, where $x \in\{n, p\}$ denotes the density of majority charge carriers in the leads, and $y^{\prime} \in\left\{n^{\prime}, p^{\prime}\right\}$ that of majority charge carriers in the cavity, where $n, n^{\prime}$ and $p$, $p^{\prime}$ are the densities of electronlike and holelike charge carriers, respectively. In a $p n^{\prime} p$ configuration, there can also be minority holes in the cavity $\left(p^{\prime}\right)$, and minority electrons in the leads $(n)$. This coexistence of electrons and holes is a direct consequence of the aforementioned band inversion. Figure 1(c) depicts the corresponding simplified band lineup for the $p n^{\prime} p$ case.

The measurement in Fig. 1(d), which contains the main findings of this work, displays $d R_{j} / d V_{\mathrm{LG}}$ as a function of $V_{\mathrm{LG}}$ and $V_{\mathrm{TG}}$ for a local gate of lithographic length $L=320 \mathrm{~nm}$. The quadrants representing the possible charge-carrier configurations indicated in the figure are separated by the experimentally determined CNPs in the leads and the cavity (horizontal and vertical dashed lines). We recognize regular oscillations in the $p n^{\prime} p$ configuration (nearly vertical stripes of alternating minima and maxima) which depend strongly on $V_{\mathrm{LG}}$, and weakly on $V_{\mathrm{TG}}$. In the other quadrants, no such oscillations appear. The observed oscillations persist up to $V_{\mathrm{LG}} \sim 1 \mathrm{~V}$ before disappearing. Concomitantly, we estimate from independent density measurements [14] that $p^{\prime}$ vanishes at $V_{\mathrm{LG}} \approx 1.1 \mathrm{~V}$, as the Fermi energy crosses the top edge of the ground state subband in the unhybridized valence band [see insets of Fig. 1(d)]. In other words, when $V_{\mathrm{LG}}>1.1 \mathrm{~V}$ we obtain a conventional electron system devoid of holes. This observation suggests that the coexistence of electrons and holes in the cavity is crucial for the emergence of the resistance oscillations. Upon closer inspection of Fig. 1(d), one can notice weak oscillatory features in the $p n^{\prime} p$ configuration also for $V_{\mathrm{LG}}>1.1 \mathrm{~V}$. Their visibility is significantly smaller than that of the oscillations found below $1.1 \mathrm{~V}$, and, as we explain later, the two are most likely not of the same origin. From now on, we concentrate on the oscillations for $V_{\mathrm{LG}}<1.1 \mathrm{~V}$.

In the $p n^{\prime} p$ configuration, $p-n$ junctions delimit the cavity. Across each junction, the density of states exhibits a local minimum as the Fermi energy transitions smoothly from below to above the hybridization gap [23]. Specifically, if a true gap exists, particles must tunnel in order to be transmitted. The $p$ - $n$ junctions can therefore act as semitransparent mirrors, trapping particles in the cavity and enabling the formation of discrete standing-wave modes. The transmission, and accordingly, the resistance, of the resulting resonant cavity is then modulated as a function of the density $n^{\prime}$ within it, with constructive and destructive interference alternating in a periodic manner. In optics, this type of interferometer is known as a Fabry-Perot étalon [24].

To better understand the nature of the resistance oscillations, we systematically investigate $p n^{\prime} p$ junctions with local gates of various lengths, both in a single device and across multiple devices, and find the oscillations to be reproducible. This robustness also holds for multiple cooldowns, with the pattern of minima and maxima hardly changing despite repeated thermal cycling. We observe that both the oscillation amplitude and the average period in $V_{\mathrm{LG}}$ decrease with increasing $L$. Furthermore, the oscillations remain unchanged if the current direction is reversed, and their amplitude also decreases with increasing

(b)
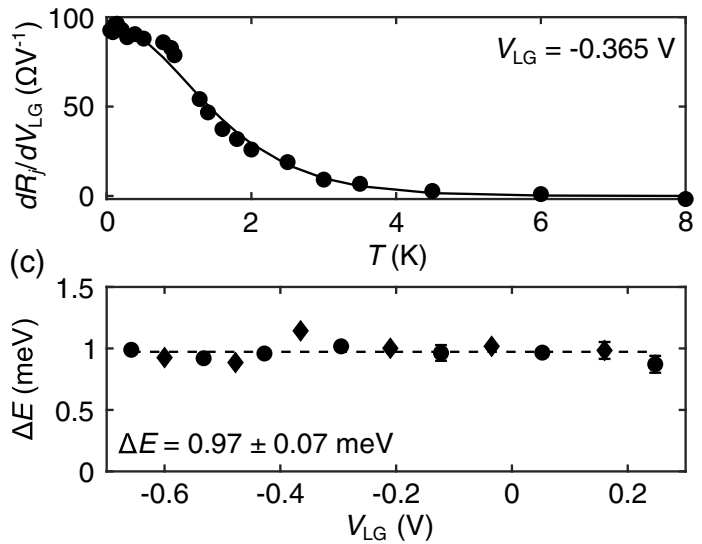

FIG. 2. (a) Temperature dependence of the resistance oscillations in $d R_{j} / d V_{\mathrm{LG}}$ in the $p n^{\prime} p$ configuration at $V_{\mathrm{TG}}=-1.8 \mathrm{~V}$ and in a reduced range in $V_{\mathrm{LG}}$, chosen so as to highlight the oscillation behavior upon changing the temperature from $50 \mathrm{mK}$ to $8 \mathrm{~K}$. Dotted lines and symbols mark local minima (circles) and maxima (diamonds) used in the analysis presented in (c). (b) Fit of the temperature dependence of the local maximum at $V_{\mathrm{LG}}=-0.365 \mathrm{~V}$, indicated by the arrow in (a), with a thermal damping function, Eq. (1). (c) Summary of the mode spacing $\Delta E$ in the cavity extracted from the local minima and maxima marked in (a). The mean and standard deviation obtained when factoring in all points is inset. The individual points have relative errors of around 5\%, such that the error bars are as large as or smaller than the point size. 
current bias. These measurements are described in the Supplemental Material [14]. A similar decrease occurs at elevated temperatures, with the oscillations completely disappearing above $\sim 4 \mathrm{~K}$. Below $\sim 500 \mathrm{mK}$ their amplitude saturates. This behavior is illustrated in Fig. 2(a).

Our experimental results are consistent with the emergence of standing wave modes in the cavity, such that the oscillations arise due to quantum interference in a twodimensional electronic analog of the Fabry-Perot étalon. The decrease in amplitude with increasing $L$ is in agreement with a finite phase coherence length. An increase in temperature leads to a broadening of the Fermi-Dirac distribution around the Fermi energy, such that the energetic interval relevant for transport expands. In the context of the cavity, this phenomenon leads to averaging over multiple adjoining standing wave modes, ultimately reducing the modulation of the resistance.

For a more quantitative analysis of the temperature dependence, we determine the oscillation amplitude as a function of temperature for the various minima and maxima, marked by circles and diamonds respectively, in Fig. 2(a). We place splines through the minima and maxima to determine the slowly varying background and subtract this background to obtain the amplitude. Figure 2(b) shows the temperature dependence of the amplitude of the maximum located at $V_{\mathrm{LG}}=-0.365 \mathrm{~V}$, as marked by the arrow in Fig. 2(a), together with a fit (solid line). The fit is given by a thermal damping function of the form

$$
A=A_{0} \frac{2 \pi^{2} k_{B} T}{\Delta E} \frac{1}{\sinh \frac{2 \pi^{2} k_{B} T}{\Delta E}},
$$

where $A_{0}$ is a constant prefactor and $\Delta E$ is the characteristic mode spacing in the cavity. We repeat the fitting procedure for the other extrema and summarize the results in Fig. 2(c). We recognize that $\Delta E$ is constant in the investigated voltage (energy) range, obtaining $\Delta E \sim 1 \mathrm{meV}$ upon taking the mean of all data points. Theoretical calculations, as discussed below, agree with this value.

\section{THEORETICAL MODELING OF FABRY-PEROT INTERFERENCE}

In order to understand the origin of the interference, we model the hybridized electron-hole system using the minimal Bernevig-Hughes-Zhang Hamiltonian [25]

$$
H=\left(\begin{array}{cc}
h(\mathbf{k}) & 0 \\
0 & h^{*}(-\mathbf{k})
\end{array}\right)
$$

where $h(\mathbf{k})=\mathcal{B} \mathbf{k}^{2} \hat{\mathbf{1}}+\left(\mathcal{M}_{0}+\mathcal{M}_{2} \mathbf{k}^{2}\right) \hat{\sigma}_{z}+\mathcal{A} k_{x} \hat{\sigma}_{x}-\mathcal{A} k_{y} \hat{\sigma}_{y}$, $\hat{\sigma}_{i}$ are Pauli matrices operating on the orbital degrees of freedom and $\mathbf{k}=\left(k_{x}, k_{y}\right)$ is the wave vector. The nonzero blocks in the Hamiltonian act on the two spin degrees of freedom separately. The parameter $\mathcal{M}_{0}$ governs the band inversion, $\mathcal{M}_{2}$ symmetrically controls the band curvatures (effective masses), $\mathcal{B}$ is a symmetry-breaking term between electron and hole bands and $\mathcal{A}$ determines the electron-hole coupling. In Fig. 3(a), we present the dispersion of the hybridized conduction band relevant for the cavity in the $p n^{\prime} p$ configuration. In the band-inverted regime, the bulk band structure has the shape of a "sombrero hat," formed due to the hybridization between the InAs conduction and $\mathrm{GaSb}$ valence bands.

We assume that both cavity and leads are infinite in the $y$ direction, such that $k_{y}$ remains a good quantum number. Scattering between the $n^{\prime}$-doped cavity and $p$-doped leads conserves $k_{y}$, such that $k_{y}=k_{y}^{\prime}$ (primed quantities refer to the cavity, unprimed ones to the leads). We therefore treat the two-dimensional scattering problem as an infinite set of one-dimensional wires with different $k_{y}$ momenta. For each $k_{y}$, we note that there are four possible scattering processes of quasiparticles that can occur at the interfaces between the cavity and the leads [Figs. 3(b) and 3(c)], distinguishing scattering between (i) alike particles-electron-toelectron and hole-to-hole (ee and $h h$ )-and (ii) different

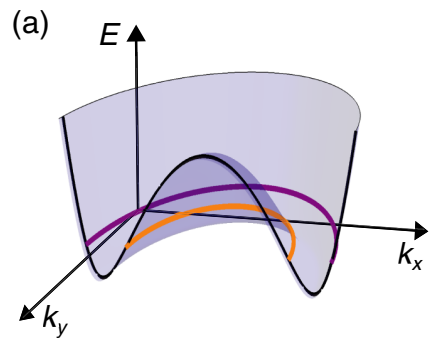

(b)

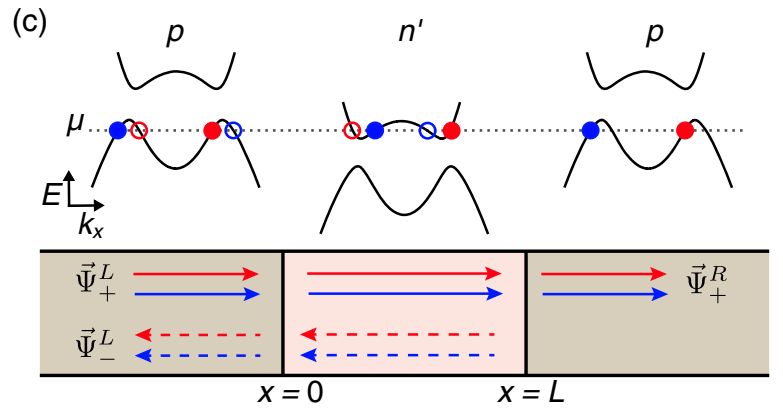

FIG. 3. (a) Sombrero-hat dispersion $E\left(k_{x}, k_{y}\right)$ of the hybridized conduction band resulting from the theoretical model, Eq. (2), as applied to the InAs/GaSb system. (b) Overview of the scattering processes $i j$ that occur within the $n^{\prime}$-doped cavity when a quasiparticle impinges onto the interface between cavity and lead. The $r_{i j}$ are reflection coefficients. (c) Upper panel: band structure in the three regions of a $p n p$ junction. The dashed horizontal line represents the electrochemical potential $\mu$. Full blue (red) circles represent holelike (electronlike) states moving to the right, while empty blue (red) circles represent holelike (electronlike) states moving to the left. Lower panel: sketch of a Fabry-Perot interferometer for a one-dimensional channel. The colors are coded according to the upper panel, with red (blue) arrows denoting holelike (electronlike) states moving to the right (solid lines) and left (dashed lines). 
particles-electron-to-hole and vice versa ( $e h$ and $h e$ ). This situation is reminiscent of optical Fabry-Perot cavities with birefringent (polarization-dependent) mirrors [26].

With the aforementioned considerations in mind, we write the scattering problem for the $p n^{\prime} p$ junction [see Fig. 3(c)] as

$$
\left(\begin{array}{c}
\vec{\Psi}_{-}^{L} \\
\vec{\Psi}_{+}^{R}
\end{array}\right)=\hat{S}\left(\begin{array}{c}
\vec{\Psi}_{+}^{L} \\
\vec{\Psi}_{-}^{R}
\end{array}\right),
$$

where $\hat{S}$ is the scattering matrix that connects the incident waves from the left lead to the transmitted waves in the right lead,

$$
\hat{S}=\left[\begin{array}{cc}
\tilde{r}^{\prime} & \tilde{t}^{\prime} \\
\tilde{t} & \tilde{r}
\end{array}\right]
$$

and $\tilde{r}, \tilde{r}^{\prime}, \tilde{t}, \tilde{t}^{\prime}$ are $2 \times 2$ matrices. We then solve the problem analytically [see the Appendix] and obtain the transmission $T\left(E^{\prime}, k_{y}\right)=\operatorname{tr} \tilde{t}^{\prime} \tilde{t}$ for each $k_{y}$ wire as a function of the Fermi energy $E^{\prime}$ in the cavity, see Fig. 4(c). The maximum possible value of $T$ is 4 , as there are 2 orbital and 2 spin degrees of freedom present. The maxima of the transmission depend only weakly on $k_{y}$ for energies close to the band bottom, near the hybridization gap. Away from the band bottom, the dependence on $k_{y}$ increases and the transmission maxima curve toward lower energies. It is crucial for our result that the wave-vector change $\Delta k_{x}$ for scattering processes $e h$ and he does not depend strongly on $k_{y}$ at energies close to the gap, see Figs. 4(a), 4(b).

Upon inspection of the reflection amplitudes $\left|r_{i j}\right|$ at the interface between the cavity and the leads [Fig. 3(b)], we identify that the $e h$ and he scattering processes dominate the transmission $T$ at energies close to the gap, see Fig. 4(d). With this in mind, we approximate the condition for constructive interference as

$$
\Delta k_{e h / h e} L=2 \pi m,
$$

where $\Delta k_{e h / h e}$ is the energy-dependent momentum transfer in the $x$ direction in the $e h$ and he scattering processes and $m \in N$ is the ordinal number of the transmission maxima [refer also to the Supplemental Material [14]]. This approximation agrees well with the calculated $T$, see Fig. 4(c).

To obtain the conductance, we integrate the calculated $T$ over all incident states with different momenta $k_{y}$,

$$
G\left(E^{\prime}\right)=\frac{2 e^{2}}{h} \frac{W}{2 \pi} \int_{-k_{F}}^{k_{F}} d k_{y} T\left(E^{\prime}, k_{y}\right)
$$

and set the resistance $R\left(E^{\prime}\right)=1 / G\left(E^{\prime}\right)$. The width of the sample in the $y$ direction is $W$ and the integration boundaries $\pm k_{F}$ are given by the Fermi wave number associated with the larger of the two Fermi circles in (a)
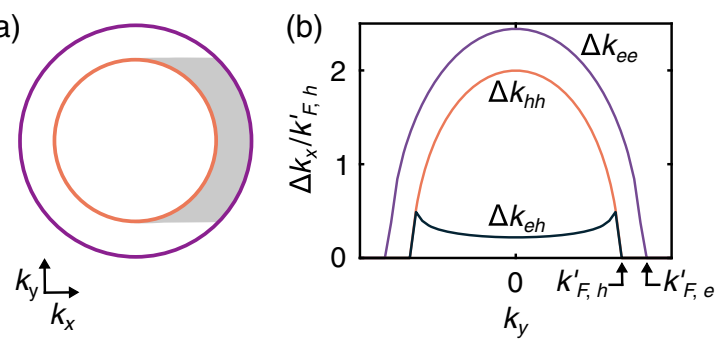

(c)

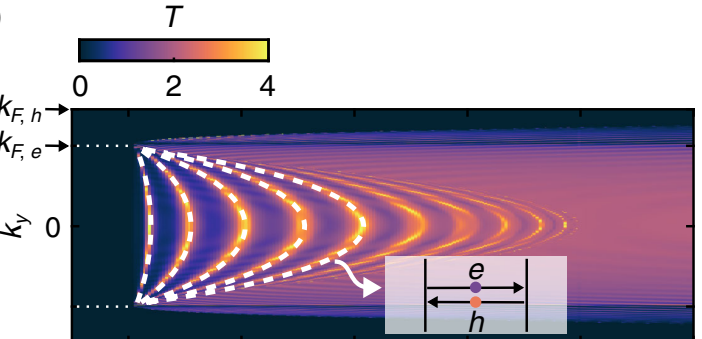

(d)

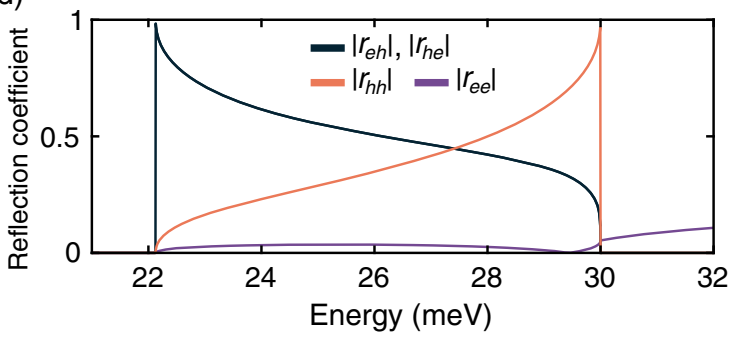

FIG. 4. (a) Two Fermi circles from Fig. 3(a) with the larger (smaller) circle being the Fermi circle of electronlike (holelike) states. The shaded region marks the momentum transfer $\Delta k_{x}$ for the $e h$ scattering processes assuming conservation of $k_{y} ; \Delta k_{x}$ does not depend strongly on $k_{y}$. (b) Momentum transfer $\Delta k_{x}$ as a function of $k_{y}$ for the various scattering processes $i j$ from Fig. 3(b). Here, $\Delta k_{e h}$ and $\Delta k_{h e}$ are identical. $k_{F, e}\left(k_{F, e}^{\prime}\right)$ and $k_{F, h}\left(k_{F, h}^{\prime}\right)$ are Fermi wave numbers of electronlike and holelike states in the leads (cavity), respectively. (c) Transmission $T$ through the $p n^{\prime} p$ junction as a function of the Fermi energy $E^{\prime}$ in the $n^{\prime}$-doped cavity and the momentum $k_{y}$ of the incident wave, calculated using a scattering matrix approach. The dashed lines denote the constructive interference condition assuming $e h$ and he scattering only [Eq. (5) and inset]. The minimum of the hybridized conduction band is located at $22.1 \mathrm{meV}$. (d) Reflection amplitudes $\left|r_{i j}\right|$ for the various scattering processes $i j$ from Fig. 3(b) plotted as a function of $E^{\prime}$ and for $k_{y}=0$.

the leads. The numerical derivative $d R / d E^{\prime}$ is shown in Fig. 5(a). Oscillations appear when the Fermi energy in the cavity lies in the energy region where the coherent $e h$ and he processes exist, indicated by the shaded area in Fig. 5(a). The energetic width of this region is approximately $8 \mathrm{meV}$, comparable to the size of the hybridization gap. Near the gap, the oscillation period grows with increasing energy. In contrast, the oscillation amplitude decays with increasing energy as the ee and $h h$ scattering processes become relevant. Once the holes in the cavity are depleted and the Fermi energy enters the region above the top of the sombrero hat, the oscillations disappear. We postulate that 
(a)

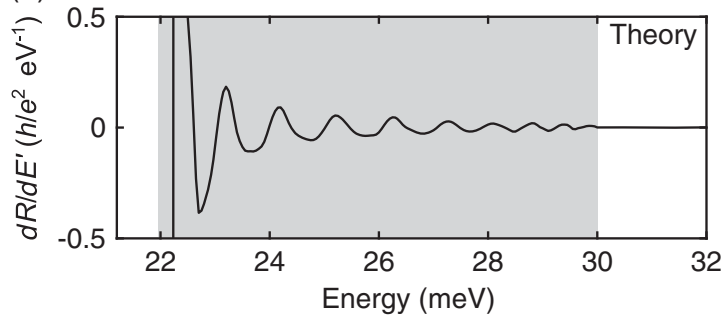

(b)

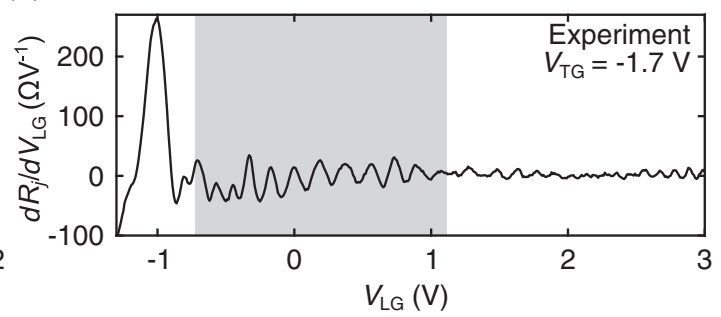

FIG. 5. (a) Differential resistance $d R / d E^{\prime}$ as a function of $E^{\prime}$ obtained from Fig. 4(c) using Eq. (6). Oscillations are present in the shaded energy region in which electrons and holes coexist. (h) Cut through Fig. 1(d) at $V_{\mathrm{TG}}=-1.7 \mathrm{~V}$, with the shaded region above the CNP marking electron and hole coexistence.

this behavior is a generic property of band structures with the shape of a sombrero hat. The interference mechanism is a result of both the selective scattering of electrons to holes and the fact that the interference condition does not vary much with $k_{y}$.

We now analyze the periodicity of the experimentally observed oscillations [Fig. 5(b)] with the theoretical insights in mind. The aforementioned features, namely the increasing period and the decreasing amplitude, both as a function of increasing energy, are present in the experiment. Furthermore, by converting gate voltage to density, and then relating the density to the wave number in the cavity, we obtain an estimate for the cavity length based on the period [14]. The estimate agrees with the lithographic gate length. It is worth emphasizing that in the case of dominant ee scattering, the oscillations would grow in amplitude with increasing electron density, or equivalently with increasing voltage. The opposite is true in the experiment, providing confirmation of the significance of electron-hole interference.

We now comment on the oscillatory features observed in the experiment in the $p n^{\prime} p$ configuration for $V_{\mathrm{LG}}>1.1 \mathrm{~V}$ in Figs. 1(d) and 5(b). Their amplitude is smaller compared to the amplitude of the Fabry-Perot oscillations and their apparent periodicity changes arbitrarily as a function of $V_{\mathrm{LG}}$. In fact, sometimes these oscillations disappear completely, as around $V_{\mathrm{LG}} \sim 2 \mathrm{~V}$, before reappearing again as a function of $V_{\mathrm{LG}}$. We have observed such features for local gate lengths $L$ which are of the order of several micrometers where we are certain that no Fabry-Perot oscillations exist. Indeed, clear and reproducible oscillations attributed to Fabry-Perot interference whose period scales inversely with $L$ and which exhibit the characteristic bending as a function of perpendicular magnetic field discussed in the next section are only present when $L$ does not exceed $\sim 1 \mu \mathrm{m}$ [see discussion on length dependence in the Supplemental Material [14] ]. Note also that the theoretical model predicts only Fabry-Perot oscillations below the top of the sombrero hat, and no oscillations above it [Fig. 5(a)], hinting at a different origin for these weaker oscillations.

We speculate the origin of the weak oscillations found above $V_{\mathrm{LG}} \sim 1.1 \mathrm{~V}$ to be mesoscopic conductance fluctuations (ballistic or diffusive). These fluctuations represent a phenomenon that competes with the Fabry-Perot interference. Once the Fabry-Perot interference disappears as holes are depleted below the local gate, the conductance fluctuations remain.

In the $n p^{\prime} n$ configuration no oscillatory features appear, even upon extension of the voltage range to more negative $V_{\mathrm{LG}}$ [14]. The origin of the lack of oscillations in this regime is currently an open question. Ideally, the interference phenomenon should appear in this configuration too. This is confirmed by our theoretical model. However, the model does not take the finite lifetime of the carriers into account. In reality, the average Drude mobility decreases when moving from the hybridized conduction band to the hybridized valence band across the CNP. This is typical of InAs/GaSb double quantum wells because the holes deep in the valence band of $\mathrm{GaSb}$ are roughly an order of magnitude heavier than the electrons deep in the conduction band of InAs [27,28]. Without invoking any particular multiband model, the average Drude mobility as determined by classical low-field magnetotransport is a combination of the electron and hole mobilities. We conjecture that the decrease in average mobility (and therefore of the elastic mean free path) is accompanied by a decrease in the phase coherence length. The combined effect is a suppression of the Fabry-Perot interference in the $n p^{\prime} n$ configuration.

\section{MAGNETIC FIELD DEPENDENCE}

We now turn to the dependence of the resistance oscillations on a perpendicular magnetic field $B$. In the experiment [Fig. 6(a)], we track the evolution of the oscillations close to the CNP as a function of $B$. Overall, lines of constant transmission (phase) are initially independent of $B$ close to $B=0$. They then bend toward lower $V_{\mathrm{LG}}$ with increasing $B$. This bending ceases thereafter as the lines straighten once again before the resistance oscillations vanish. Comparing the $d R_{j} / d V_{\mathrm{LG}}$ signal at $B=0$ and at $B=0.25 \mathrm{~T}$, we recognize that minima and maxima are interchanged. Such a shift of half a period is equivalent to a phase shift of $\pi$. 

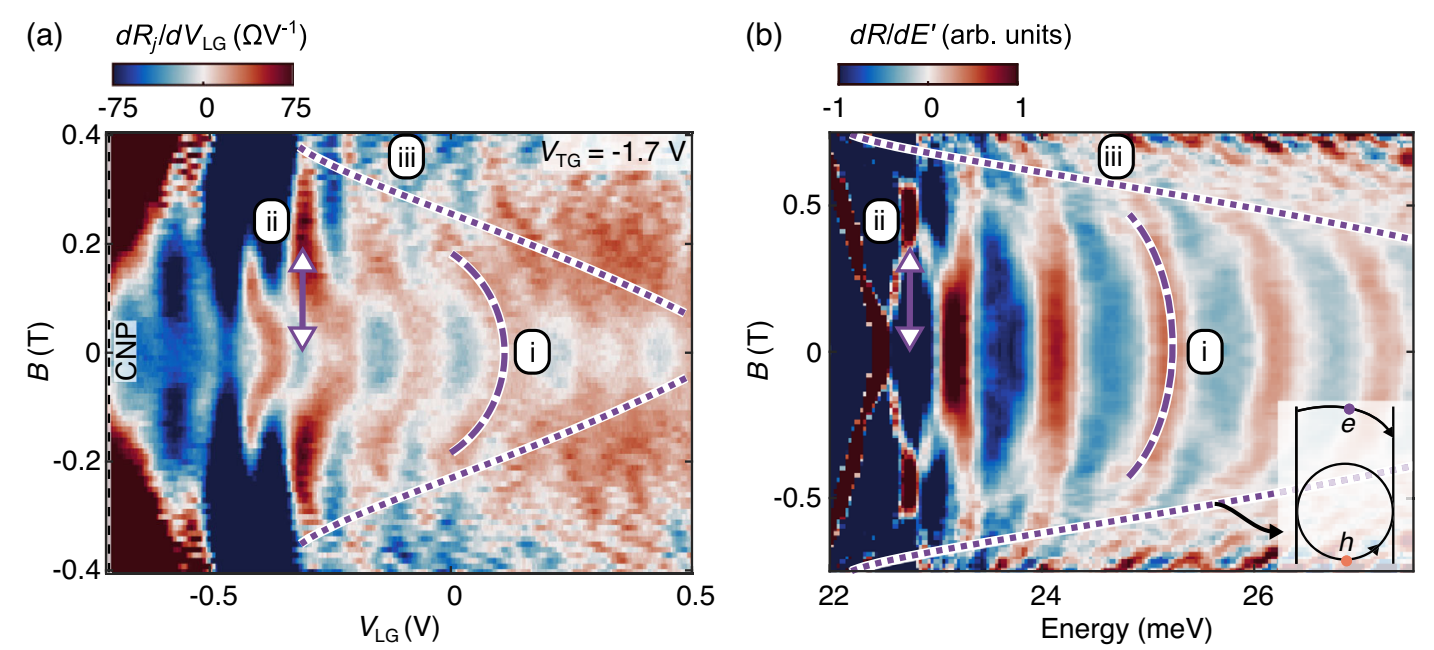

FIG. 6. (a) Color map depicting the differential junction resistance $d R_{j} / d V_{\mathrm{LG}}$ as a function of $V_{\mathrm{LG}}$ and $B$ at $V_{\mathrm{TG}}=-1.7 \mathrm{~V}$ and $1.3 \mathrm{~K}$. (b) Calculated dependence of $d R / d E^{\prime}$ on the Fermi energy $E^{\prime}$ in the cavity and $B$. The inset shows schematic trajectories for electronlike and holelike states at the critical magnetic field $B_{c}$ of the holelike states, represented in both (a) and (b) by the dotted lines. Dashed lines and arrows indicate features common to both experiment and calculation, discussed under points (i)-(iii) in the main text.

Placing model (2) on a lattice [29], we numerically calculate the expected magnetic field dependence, see Fig. 6(b). Crucially, opposite spin states possess different responses to the magnetic field. The resulting plot bears similarities with the experimental measurement, and qualitatively captures all striking observations, namely (i) the qualitative bending of the lines, (ii) the exchange of minima and maxima, and (iii) the energy-dependent (gate-voltage dependent) critical magnetic field above which oscillations are no longer present. From the model analysis, we conclude that (i) highlights the holelike nature of the cavity states, (ii) is related to the spin splitting of the initially degenerate standing wave modes in the cavity, and (iii) is a result of the decreasing density of holelike states (see below). Note that the minimal model (2) does not include several complications present in the experiment, such as band anisotropy, additional spin-orbit coupling terms, a density-dependent band overlap, and the fact that the electrostatics of the device change as a function of the gate voltages. These effects may conspire to produce the quantitative differences between the two plots in Fig. 6 .

Semiclassically, we expect the interference phenomenon to be suppressed with increasing magnetic field as the cyclotron radius $r_{c}=\hbar k_{F}^{\prime} / e B$ decreases below a threshold related to the length $L$ of the cavity, where $k_{F}^{\prime}$ is the Fermi wave number in the cavity. Namely, when $2 r_{c}=L$ at a critical field $B_{c}$, quasiparticles cannot traverse the cavity any longer. As the holelike states have smaller $k_{F}^{\prime}$ than the electronlike states, their $B_{c}$ occurs earlier, see dotted lines and the inset in Fig. 6(b). While it is not straightforward to determine $B_{c}$ in the experiment [the corresponding lines in Fig. 6(a) are guides to the eye only], in the model analysis the oscillations essentially disappear when this condition is fulfilled for the holelike states. Therefore, we conclude that in the $p n^{\prime} p$ configuration the minority holes in the cavity decisively govern the interference dynamics in the presence of a magnetic field and not the majority electrons.

\section{OUTLOOK}

Our results set the stage for engineering electron optics phenomena in a variety of materials that feature band inversion and hybridization, or equivalently, a sombrero-hat dispersion. Examples include systems with strong Rashba spin-orbit interaction [30], such as the $\mathrm{HgTe} / \mathrm{CdTe}$ quantum spin Hall insulator system [31], two-dimensional monochalcogenides like GaSe [32], transition metal dichalcogenides like $\mathrm{WTe}_{2}$ [33] and three-dimensional topological insulators, such as those based on bismuth [34]. We note that the coherent oscillations in the resistance are also dependent on the geometry of the cavity, most notably on the cavity length. Although the aforementioned systems have a sombrero-hat dispersion, it is important that the length of the cavity is large enough such that several modes fit inside the energy region where the electronlike and holelike states are present. On the other hand, the cavity needs to be small enough such that the separation of the oscillations can be experimentally resolved. After briefly examining the band structures of said systems, we believe that they too should feature Fabry-Perot interference mediated by electron-to-hole scattering in the bandinverted regime, provided their material quality guarantees a sufficiently large phase coherence length. A more thorough exposition of the generality of the newly discovered interference mechanism is explicated in the Supplemental Material [14].

We also predict that single $p$ - $n$ junctions in the above materials can exhibit focusing of electrons and holes due to electron-to-hole scattering, thus enabling the 
implementation of a range of devices such as Veselago lenses [8,9] and beam splitters [11]. In the particular case of InAs/GaSb, the electrostatic tunability with back and front gates is advantageous for precise control of the band structure [28] and hence the interference phenomenon. Compatibility with standard large-scale semiconductor processing techniques enables the straightforward realization of networks of interferometers, which is favorable for upscaling.

\section{ACKNOWLEDGMENTS}

We thank P. Rickhaus for helpful discussions. This work was supported by the ETH FIRST laboratory. We acknowledge financial support from the Swiss National Science Foundation through the Division II, through the National Center of Competence in Research on Quantum Science and Technology (NCCR QSIT) and through Grant No. PP00P2_163818.

\section{APPENDIX: SCATTERING MATRIX}

We employ a scattering matrix approach to calculate the transmission through the device. Because of the different positions of the Fermi energy in the cavity and the leads, effective barriers emerge at the cavity-lead interfaces, entailing scattering of waves at these barriers, see Fig. 3(c). As a result of the sombrero-hat band structure, for each energy located within the region of band overlap there exists a region of momenta $k_{y}$ where two incident waves are present, one of them being electronlike and the other holelike [Fig. 3(c)].

We work in the limit of infinitely sharp $p-n$ interfaces, although preliminary studies show that the oscillations are also present if the $p-n$ interfaces are broadened. In Fig. 3(c), the scattering problem for one $k_{y}$ channel is illustrated. At every interface, the scattering matrix is equal to

$$
\hat{S}_{L / R}=\left[\begin{array}{cc}
r_{L / R}^{\prime} & t_{L / R}^{\prime} \\
t_{L / R} & r_{L / R}
\end{array}\right] \text {, }
$$

where the subscript $L(R)$ denotes the left (right) interface and $r, r^{\prime}, t, t^{\prime}$ are $2 \times 2$ matrices describing reflection and transmission amplitudes for electronlike and holelike waves impinging on the interface from the left $(r, t)$ and the right $(r, t)$ with the following structure:

$$
\begin{array}{rlr}
r=\left[\begin{array}{ll}
r_{e e} & r_{e h} \\
r_{h e} & r_{h h}
\end{array}\right], & t=\left[\begin{array}{ll}
t_{e e} & t_{e h} \\
t_{h e} & t_{h h}
\end{array}\right], \\
r^{\prime}=\left[\begin{array}{ll}
r_{e e}^{\prime} & r_{e h}^{\prime} \\
r_{h e}^{\prime} & r_{h h}^{\prime}
\end{array}\right], & t^{\prime}=\left[\begin{array}{ll}
t_{e e}^{\prime} & t_{e h}^{\prime} \\
t_{h e}^{\prime} & t_{h h}^{\prime}
\end{array}\right] .
\end{array}
$$

We can write down the scattering equations for the $p n^{\prime} p$ junction,

$$
\begin{gathered}
\left.\left(\begin{array}{c}
\vec{\Psi}_{-}^{L} \\
\vec{\Psi}_{+}^{C}
\end{array}\right)\right|_{x=0}=\left.\hat{S}_{L}\left(\begin{array}{c}
\vec{\Psi}_{+}^{L} \\
\vec{\Psi}_{-}^{C}
\end{array}\right)\right|_{x=0}, \\
\left(\begin{array}{c}
\vec{\Psi}_{-}^{C}(x=0) \\
\vec{\Psi}_{+}^{C}(x=L)
\end{array}\right)=\hat{T}\left(\begin{array}{l}
\vec{\Psi}_{-}^{C}(x=L) \\
\vec{\Psi}_{+}^{C}(x=0)
\end{array}\right), \\
\left.\left(\begin{array}{c}
\vec{\Psi}_{-}^{C} \\
\vec{\Psi}_{+}^{R}
\end{array}\right)\right|_{x=L}=\left.\hat{S}_{R}\left(\begin{array}{l}
\vec{\Psi}_{+}^{C} \\
\vec{\Psi}_{-}^{R}
\end{array}\right)\right|_{x=L},
\end{gathered}
$$

where $C$ denotes the cavity and the subscripts +/denote waves moving to the right or left, i.e., waves with positive or negative velocities. In general, $\vec{\Psi}_{ \pm}^{j}=$ $\left[\psi^{j}\left( \pm\left|k_{e}\right|\right) \psi^{j}\left(\mp\left|k_{h}\right|\right)\right]^{T}$ is a two-component vector with spinor components that denote waves with electronlike dispersion [marked with red circles in Fig. 3(c)] and waves with holelike dispersion [blue circles in Fig. 3(c)]. The matrix $\hat{T}$ assigns the dynamical phase for the waves due to their free propagation, and is equal to

$\hat{T}=\left[\begin{array}{cccc}e^{i\left|k_{e}\right| L} & 0 & 0 & 0 \\ 0 & e^{-i\left|k_{h}\right| L} & 0 & 0 \\ 0 & 0 & e^{i\left|k_{e}\right| L} & 0 \\ 0 & 0 & 0 & e^{-i\left|k_{h}\right| L}\end{array}\right] \equiv\left[\begin{array}{cc}\hat{\phi} & 0 \\ 0 & \hat{\phi}\end{array}\right]$.

Now that we have set up the scattering problem in terms of scattering processes at each junction individually, we can proceed in determining the total transmission through the device.

The equations that connect the incident states from the left lead to the transmitted waves in the right lead are given by Eq. (4). In the experiment, current is injected only from the left lead and so we set $\vec{\Psi}_{-}^{R}=0$. The final solution for the waves transmitted in the right lead, $\vec{\Psi}_{+}^{R}$, is $\vec{\Psi}_{+}^{R}=\tilde{t}_{+}^{L}$, and hence the total transmission is $T\left(E^{\prime}, k_{y}\right)=\operatorname{tr} \tilde{t} \dot{t}$. The matrix $\tilde{t}$ can be obtained from the matrices $\hat{S}_{L}, \hat{S}_{R}$, and $\hat{\phi}$. After taking into account the time-reversal symmetry, $\hat{S}_{L / R}^{T}=\hat{S}_{L / R}$, and inversion symmetry around the middle of the cavity, at $x=L / 2, \hat{S}_{R}\left(E^{\prime}, k_{y}\right)=\sigma_{x} \hat{S}_{L}\left(E^{\prime},-k_{y}\right) \sigma_{x}$, where $\sigma_{x}$ is a Pauli matrix, we obtain

$$
\tilde{t}=\bar{t}^{T} \hat{\phi}\left(\hat{\mathbf{1}}_{2 \times 2}-r \hat{\phi} \bar{r} \hat{\phi}\right)^{-1} t,
$$

with $\left\{\bar{r}\left(k_{y}\right), \bar{t}\left(k_{y}\right)\right\}=\left\{r\left(-k_{y}\right), t\left(-k_{y}\right)\right\}$.

[1] W. Liang, M. Bockrath, D. Bozovic, J. H. Hafner, M. Tinkham, and H. Park, Fabry-Perot Interference in a Nanotube Electron Waveguide, Nature (London) 411, 665 (2001).

[2] B. J. van Wees, L. P. Kouwenhoven, C. J. P. M. Harmans, J. G. Williamson, C. E. Timmering, M. E. I. Broekaart, 
C. T. Foxon, and J. J. Harris, Observation of ZeroDimensional States in a One-Dimensional Electron Interferometer, Phys. Rev. Lett. 62, 2523 (1989).

[3] Y. Ji, Y. Chung, D. Sprinzak, M. Heiblum, D. Mahalu, and H. Shtrikman, An Electronic Mach-Zehnder Interferometer, Nature (London) 422, 415 (2003).

[4] A. F. Young and P. Kim, Quantum Interference and Klein Tunnelling in Graphene Heterojunctions, Nat. Phys. 5, 222 (2009).

[5] L. C. Campos, A. F. Young, K. Surakitbovorn, K. Watanabe, T. Taniguchi, and P. Jarillo-Herrero, Quantum and Classical Confinement of Resonant States in a Trilayer Graphene Fabry-Perot Interferometer, Nat. Commun. 3, 1239 (2012).

[6] A. Varlet, M.-H. Liu, V. Krueckl, D. Bischoff, P. Simonet, K. Watanabe, T. Taniguchi, K. Richter, K. Ensslin, and T. Ihn, Fabry-Perot Interference in Gapped Bilayer Graphene with Broken Anti-Klein Tunneling, Phys. Rev. Lett. 113, 116601 (2014).

[7] J. Spector, H. L. Stormer, K. W. Baldwin, L. N. Pfeiffer, and K.W. West, Electron Focusing in Two-Dimensional Systems by Means of an Electrostatic Lens, Appl. Phys. Lett. 56, 1290 (1990).

[8] V. V. Cheianov, V. Fal'ko, and B. L. Altshuler, The Focusing of Electron Flow and a Veselago Lens in Graphene p-n Junctions, Science 315, 1252 (2007).

[9] G.-H. Lee, G.-H. Park, and H.-J. Lee, Observation of Negative Refraction of Dirac Fermions in Graphene, Nat. Phys. 11, 925 (2015).

[10] C.-H. Park, Y.-W. Son, L. Yang, M. L. Cohen, and S. G. Louie, Electron Beam Supercollimation in Graphene Superlattices, Nano Lett. 8, 2920 (2008).

[11] P. Rickhaus, P. Makk, M.-H. Liu, K. Richter, and C. Schnenberger, Gate Tuneable Beamsplitter in Ballistic Graphene, Appl. Phys. Lett. 107, 251901 (2015).

[12] S. Chen, Z. Han, M. M. Elahi, K. M. M. Habib, L. Wang, B. Wen, Y. Gao, T. Taniguchi, K. Watanabe, J. Hone, A. W. Ghosh, and C. R. Dean, Electron Optics with p-n Junctions in Ballistic Graphene, Science 353, 1522 (2016).

[13] H. Kroemer, The 6.1 $\AA$ Family (InAs, GaSb, AlSb) and Its Heterostructures: A Selective Review, Physica (Amsterdam) 20E, 196 (2004).

[14] See Supplemental Material at http://link.aps.org/ supplemental/10.1103/PhysRevX.10.031007 for more details.

[15] C. Liu, T. L. Hughes, X.-L. Qi, K. Wang, and S.-C. Zhang, Quantum Spin Hall Effect in Inverted Type-II Semiconductors, Phys. Rev. Lett. 100, 236601 (2008).

[16] K. Suzuki, Y. Harada, K. Onomitsu, and K. Muraki, Edge Channel Transport in the InAs/GaSb Topological Insulating Phase, Phys. Rev. B 87, 235311 (2013).

[17] L. Du, I. Knez, G. Sullivan, and R.-R. Du, Robust Helical Edge Transport in Gated InAs/GaSb Bilayers, Phys. Rev. Lett. 114, 096802 (2015).

[18] V. S. Pribiag, A. J. A. Beukman, F. Qu, M. C. Cassidy, C. Charpentier, W. Wegscheider, and L. P. Kouwenhoven, Edge-Mode Superconductivity in a Two-Dimensional Topological Insulator, Nat. Nanotechnol. 10, 593 (2015).

[19] M. Altarelli, Electronic Structure and SemiconductorSemimetal Transition in InAs-GaSb Superlattices, Phys. Rev. B 28, 842 (1983).
[20] M. Lakrimi, S. Khym, R. J. Nicholas, D. M. Symons, F. M. Peeters, N. J. Mason, and P. J. Walker, Minigaps and Novel Giant Negative Magnetoresistance in InAs/GaSb Semimetallic Superlattices, Phys. Rev. Lett. 79, 3034 (1997).

[21] M. J. Yang, C. H. Yang, B. R. Bennett, and B. V. Shanabrook, Evidence of a Hybridization Gap in "Semimetallic" InAs/ GaSb Systems, Phys. Rev. Lett. 78, 4613 (1997).

[22] L. J. Cooper, N. K. Patel, V. Drouot, E. H. Linfield, D. A. Ritchie, and M. Pepper, Resistance Resonance Induced by Electron-Hole Hybridization in a Strongly Coupled InAs/GaSb/AlSb Heterostructure, Phys. Rev. B 57, 11915 (1998).

[23] M. Karalic, C. Mittag, T. Tschirky, W. Wegscheider, K. Ensslin, and T. Ihn, Lateral p-n Junction in an Inverted InAs/GaSb Double Quantum Well, Phys. Rev. Lett. 118, 206801 (2017).

[24] B. E. A. Saleh and M. C. Teich, Fundamentals of Photonics (John Wiley \& Sons, 2019), https://www.wiley.com/en-us/ Fundamentals+of+Photonics $\% 2 \mathrm{C}+2+$ Volume+Set $\% 2 \mathrm{C}+3 \mathrm{rd}+$ Edition-p-9781119506874.

[25] B. A. Bernevig, T. L. Hughes, and S.-C. Zhang, Quantum Spin Hall Effect and Topological Phase Transition in HgTe Quantum Wells, Science 314, 1757 (2006).

[26] M. Okada and S. Ieiri, Electronic Tuning of Dye Lasers by an Electrooptic Birefringent Fabry-Perot Etalon, Opt. Commun. 14, 4 (1975).

[27] L. S. Kim, H. D. Drew, H. Munekata, L. L. Chang, and L. Esaki, Electron and Hole Cyclotron Resonance in Semimetallic GaSb/InAs/GaSb Quantum Wells, Solid State Commun. 66, 873 (1988).

[28] F. Qu, A. J. A. Beukman, S. Nadj-Perge, M. Wimmer, B.-M. Nguyen, W. Yi, J. Thorp, M. Sokolich, A. A. Kiselev, M. J. Manfra, C. M. Marcus, and L. P. Kouwenhoven, Electric and Magnetic Tuning Between the Trivial and Topological Phases in InAs/GaSb Double Quantum Wells, Phys. Rev. Lett. 115, 036803 (2015).

[29] C. W. Groth, M. Wimmer, A. R. Akhmerov, and X. Waintal, Kwant: A Software Package for Quantum Transport, New J. Phys. 16, 063065 (2014).

[30] Y. A. Bychkov and E. I. Rashba, Properties of a $2 D$ Electron Gas with Lifted Spectral Degeneracy, JETP Lett. 39, 78 (1984), http://www.jetpletters.ac.ru/ps/1264/ article_19121.shtml.

[31] G. M. Minkov, A. V. Germanenko, O. E. Rut, A. A. Sherstobitov, S. A. Dvoretski, and N. N. Mikhailov, TwoDimensional Semimetal in a Wide HgTe Quantum Well: Magnetotransport and Energy Spectrum, Phys. Rev. B 88, 155306 (2013).

[32] X. Li, M.-W. Lin, A. A. Puretzky, J. C. Idrobo, C. Ma, M. Chi, M. Yoon, C. M. Rouleau, I. I. Kravchenko, D. B. Geohegan, and K. Xiao, Controlled Vapor Phase Growth of Single Crystalline, Two-Dimensional GaSe Crystals with High Photoresponse, Sci. Rep. 4, 5497 (2014).

[33] X. Qian, J. Liu, L. Fu, and J. Li, Quantum Spin Hall Effect in Two-Dimensional Transition Metal Dichalcogenides, Science 346, 1344 (2014).

[34] H. Zhang, C.-X. Liu, X.-L. Qi, X. Dai, Z. Fang, and S.-C. Zhang, Topological Insulators in $\mathrm{Bi}_{2} \mathrm{Se}_{3}, \mathrm{Bi}_{2} \mathrm{Te}_{3}$ and $\mathrm{Sb}_{2} \mathrm{Te}_{3}$ with a Single Dirac Cone on the Surface, Nat. Phys. 5, 438 (2009). 